\title{
Factores Asociados a la Disposición por el Teletrabajo entre Docentes Universitarios
}

\section{FACTORS ASSOCIATED WITH THE DISPOSITION FOR TELEWORK AMONG UNIVERSITY TEACHERS}

Omar Tapasco Alzate ${ }^{1}$, Jaime Giraldo Garcia ${ }^{2}$

1. Profesor Asistente Universidad de Caldas, Manizales, Colombia; Profesor Catedrático Universidad Nacional de Colombia sede Manizales, Colombia.

2. Profesor Titular Universidad Nacional de Colombia sede Manizales, Colombia.

\section{RESUMEN}

Son múltiples los beneficios reportados alrededor del mundo con referencia a la adopción del teletrabajo, y es ampliamente recomendada su implementación para actividades relacionadas con la gestión del conocimiento y la información. Pero es muy escasa la investigación adelantada al respecto en el sector educativo. La presente investigación se propuso determinar qué factores inciden con la disposición hacia la adopción del teletrabajo entre profesores universitarios. Se aplicó una encuesta entre 118 profesores de universidades públicas y privadas del sector del Eje Cafetero-Colombia en donde se recolectó información relacionada con aspectos individuales, actividades tele-trabajables, percepción y actitud hacia el uso de TIC, aptitud para el manejo de TIC, y aspectos relacionados con su entorno laboral. Se aplicaron pruebas de independencia, análisis de correspondencias múltiples y regresión logística. Se detecta una alta disposición hacia la adopción del teletrabajo entre profesores universitarios. Entre los factores asociados hacia el interés por teletrabajar surgen principalmente los relacionados con los aspectos aptitudinales y actitudinales hacia el uso de las TIC en las actividades laborales, mientras que por el contrario, los aspectos individuales, el entorno laboral y el tipo de actividades realizadas presentan muy poca incidencia en la disposición a teletrabajar.

(Tapasco 0, Giraldo J, 2016. Factores Asociados a la Disposición por el Teletrabajo entre Docentes Universitarios. Cienc Trab. May-Ago; 18 [56]: 87-93).

Palabras claves: TELETRABAJO, TECNOLOGÍAS DE LA COMUNICACIÓN Y LA INFORMACIÓN TIC, PROFESORES UNIVERSITARIOS, ANÁLISIS DE CORRESPONDENCIAS MÚLTIPLES, REGRESIÓN LOGÍSTICA.

\section{ABSTRACT}

Multiple benefits are reported around the world with reference to the adoption of telework, and is widely recommended its implementation in activities related to knowledge management and information. But there is little research in the education sector. This research aims to determine what factors affect the disposition towards adoption of telework among university teachers. A survey was done among 118 teachers from public and private universities of a region of Colombia where related information with individual aspects, teleworking activities, perceptions and attitudes towards the use of ICT, aptitude for the management of ICT, and aspects related to their work environment was collected. Tests of independence, multiple correspondence analysis and logistic regression were applied. A high disposition towards the adoption of telework among academics is detected. Among the factors associated to the interest in telecommuting arise primarily those related to the aptitudinal and attitudinal aspects to the use of ICT in work activities, while on the contrary, the individual aspects, the working environment and the type of activities have very little impact on the willingness to telework.

Keywords: TELEWORK, INFORMATION TECHNOLOGY AND COMMUNICATIONS ICT, UNIVERSITY TEACHERS, MULTIPLE CORRESPONDENCE ANALYSIS, LOGISTIC REGRESSION.

\section{INTRODUCCIÓN}

El desarrollo acelerado de las Tecnologías de la Información y la Comunicación (TIC) ha generado fuertes cambios en la sociedad actual en todos los ámbitos y uno de ellos es precisamente el relacionado con el aspecto laboral. Es así como se han abierto las

\footnotetext{
Correspondencia / Correspondence

Omar Tapasco Alzete

Manizales, Caldas, Colombia.

Tel: +57 68879300

e-mail: oatapascoa@unal.edu.co

Recibido: / Aceptado:
}

posibilidades para que los trabajadores, haciendo uso de dichas herramientas, puedan realizar gran parte de su labor sin necesidad de movilizarse hasta las instalaciones de las organizaciones, lo que se conoce como otra modalidad de trabajo denominada teletrabajo. Y son múltiples los beneficios reportados, tanto para el empleado como para las organizaciones y la sociedad en general por la adopción de esta modalidad laboral. Entre ellas se tiene que la práctica del teletrabajo afecta positivamente el desempeño de la organización, disminuyendo el ausentismo laboral ${ }^{1}$ y las intenciones de cambiar de $\operatorname{trabajo}^{2,3}$ y reiterativamente se hace mención sobre la evidencia hallada en el incremento en la productividad del trabajador. ${ }^{2,3,4} \mathrm{Y}$ unido al hecho de que los teletrabajadores alcanzan una mayor autonomía y flexibilidad, se ha reportado una reducción significativa del estrés, el aburrimiento y la fatiga relacionada a las actividades laborales, así 
como una mejor conciliación entre la vida laboral y familiar del teletrabajador. $^{4,5}$ Igualmente, se ha encontrado un incremento en la satisfacción de los empleados y un mayor compromiso de los mismos con la organización. ${ }^{2,5}$

Adicionalmente, el teletrabajo ofrece una alternativa para cuando se presentan eventos inesperados, tales como huelgas de transporte, o condiciones severas de tiempo, o desastres naturales o epidemias. ${ }^{2}$ Entre los beneficios evidenciados para la sociedad se hace hincapié en la reducción del tráfico en las grandes ciudades, lo cual genera un impacto positivo en la movilidad ${ }^{6}$, disminuyendo las emisiones contaminantes al aire y los niveles de ruido. ${ }^{1}$ También facilita el acceso al mercado laboral de personas con restricciones de movilización, bien sea porque presentan algún tipo de discapacidad física o porque dentro de sus labores cotidianas está el cuidado permanente de familiares. ${ }^{7}$ Por el contrario, entre las principales desventajas en su implementación se destacan la sensación de aislamiento y la pérdida de visibilidad del teletrabajador hacia la organización ${ }^{7}$, conductas adictivas al trabajo y otras problemáticas asociadas al riesgo psicosocial. ${ }^{8} \mathrm{Y}$ entre los desafíos que se desprenden de la implementación del teletrabajo están el de monitorear y evaluar el desempeño basado en resultados más allá de la presencia física del empleado $^{9}$ y el establecimiento de una normativa clara que proporcione una regulación legal y jurídica, faltante aun en muchos países latinoamericanos. ${ }^{8}$

De manera particular, en el ámbito de la educación la integración de las TIC a las instituciones educativas genera un aumento de la transferencia de los aprendizajes a la sociedad y el desarrollo de espacios flexibles para el aprendizaje, agiliza las actividades administrativas, potencia la autonomía personal y el desarrollo del trabajo colaborativo y cooperativo, entre otros. ${ }^{10}$ En el mismo sentido, altos niveles de apoyo en el teletrabajo para la práctica docente están asociadas con altos niveles de productividad y satisfacción, bajos niveles de fatiga física y emocional, y una reducción en el estrés laboral, la frustración y la sobrecarga laboral. ${ }^{11}$

Pero a pesar de los beneficios evidenciados, las dinámicas de implementación del teletrabajo a nivel mundial han estado por debajo de las expectativas, lo que se acentúa en países en vía de desarrollo donde el crecimiento en la adopción de esta modalidad laboral ha sido realmente muy lento. ${ }^{3}$ Se tiene, además, que la gran mayoría de las investigaciones que a la fecha han abordado el estudio del teletrabajo lo han hecho en el sector empresarial y muy pocas en instituciones de educación superior, aun sabiendo que es de común conocimiento que los profesores teletrabajan algunas veces, y que desde la perspectiva de la realización del trabajo no presencial, y considerando que el trabajo académico ha sido tradicionalmente evaluado por resultados, la carencia de presencia física puede ser un problema menor. ${ }^{9}$

El presente estudio está direccionado hacia la determinación de los aspectos potencialmente influyentes en la disposición que tienen los profesores de educación superior hacia adoptar el teletrabajo para algunas de sus actividades académicas en donde no sea primordial la presencialidad. Pero dicha decisión de adoptar el teletrabajo está influenciada por una variedad de elementos tecnológicos, institucionales y organizacionales, así como por características individuales y de percepción y actitud hacia el uso de las TIC. ${ }^{12}$ Es claro que las principales influencias inhibitorias para la adopción y la difusión del teletrabajo están relacionadas con factores humanos y organizacionales más que con factores tecnológicos, sin desconocer la importancia de la confianza y la autonomía del trabajo, el cambio administrativo, así como el disponer de una infraestructura tecnológica necesaria para la adopción y práctica del teletrabajo. ${ }^{13}$ En el presente estudio, los factores reportados como influyentes en las dinámicas de adopción del teletrabajo se han agrupado en las siguientes dimensiones: características individuales, actividades tele-trabajables, aspectos actitudinales y de percepción hacia las TIC, aptitud en el manejo de las herramientas TIC, y aspectos relacionados con la disponibilidad de recursos al interior de las instituciones educativas.

\section{Características Individuales}

Aunque los teletrabajadores no nacen, se hacen, hay ciertas características personales afines a una buena labor del teletrabajador, tales como la auto-disciplina y la habilidad de trabajar independientemente. ${ }^{14}$ En relación con los aspectos generacionales, investigadores ${ }^{15}$ reportan que existen diferencias en la percepción hacia el teletrabajo entre personas de la generación de "los millennials", es decir, nacidos en 1981 en adelante, con personas de otras generaciones, encontrando que las personas de dicha generación dan una importancia superior a la autonomía, al balance trabajo-vida y evidencian mejores habilidades computacionales, matizado por la escolaridad de la persona y sus competencias computacionales. Otros investigadores ${ }^{16,17}$ plantean que a las mujeres se les presentan menos opciones para teletrabajar, pero que son más proclives a escoger el teletrabajo cuando se les presenta la oportunidad. De igual forma, se sabe que la preferencia por el teletrabajo también está positivamente influenciado por el número de hijos, el no vivir solo, el ser hombre y el ser joven. ${ }^{14,18}$ En igual sentido se reporta que la flexibilidad y la libertad en el horario de trabajo conducen a la realización del trabajo en los momentos más creativos y productivos, lo que para algunos trabajadores no corresponde a las horas de trabajo regular. ${ }^{2}$

\section{Actividades Tele-Trabajables}

Algunas tareas son dependientes del sitio de trabajo y consecuentemente no se ajustan para ser tele-trabajables. Sin embargo, en la actualidad, un creciente porcentaje de la fuerza laboral son trabajadores de la información y el conocimiento, por lo que los trabajadores no son dependientes de lugar de trabajo. ${ }^{12}$ Factores tales como el uso frecuente del computador, el grado de escolaridad y las habilidades en el uso de las TIC muestran asociación con la preferencia por teletrabajar. ${ }^{18}$ Puntualmente, la labor docente avizorada desde las distintas dimensiones del quehacer universitario, como son la docencia, la investigación y la extensión, contempla la realización de múltiples actividades que no requieren de forma obligatoria de su presencia en la institución, tales como la preparación de clases, la elaboración y calificación de actividades evaluativas, reuniones con colegas, trabajo investigativo, actividades de extensión universitaria, entre otros, por lo que de manera parcial se evidencia una afinidad entre el quehacer del profesor universitario y el teletrabajo.

\section{Aspectos Actitudinales}

Dado que el teletrabajo es una modalidad de trabajo relativamente nueva, su adopción puede relacionarse con un riesgo hacia la estabilidad laboral, por lo que muchos trabajadores quizás no estén dispuestos a aventurarse a tales riesgos. Por el contrario, una insatisfacción por las disposiciones laborales actuales o por la baja calidad de tiempo que se pasa con las propias familias puede tener una influencia positiva hacia la adopción del teletrabajo. ${ }^{12}$ En el ámbito académico, se observa en la actualidad que los estudiantes que ingresan a la universidad están más familiarizados con formatos de comunicación a través de herramientas de aprendizaje 
social, tales como Facebook, Twitter y WhatsApp, y esperan que estas sean replicadas en el salón de clases, lo que conduce a que los métodos de enseñanza tradicional lleguen a ser cuestionados. Por lo que, consecuentemente, el personal académico está encarando distintos retos para la inmersión en los nuevos ambientes de aprendizaje, lo cual adiciona complejidad y presión cuando ellos enseñan a estudiantes con una diversidad incrementada. ${ }^{19}$

\section{Aspectos Aptitudinales}

La literatura reporta que el interés por teletrabajar está positivamente relacionado con la familiaridad con las nuevas tecnologías $^{16}$, por lo que la adopción del teletrabajo será más fácil si las TIC están ya siendo usadas en la organización. En el ámbito de la docencia universitaria, se evidencia una brecha generacional entre profesores y estudiantes en términos del uso de internet, intereses y habilidades. ${ }^{20}$ Por lo que se hace necesario que las instituciones de educación superior diseñen cursos de formación que faciliten la incorporación de las TIC en la práctica docente ${ }^{21}$ y que proporcionen un adecuado desarrollo profesional, mediante el entrenamiento y el apoyo administrativo a los académicos, conducentes a incrementar su conocimiento en la compleja interrelación entre tecnología, pedagogía y contenido cognitivo en sus disciplinas. ${ }^{19}$ De igual forma, los profesores también tendrán que procurar por sí mismos estar en constante capacitación, no sólo con todo aquello que se relacione con la tecnología en general, sino también con respecto a los medios que realmente requiera.

\section{Entorno Institucional}

Para que el teletrabajo pueda ser exitosamente implementado será necesario que un conjunto mínimo de requerimientos tecnológicos y organizacionales sean cumplidos, dado que una organización con una inadecuada tecnología conduciría a los teletrabajadores a experimentar un proceso de trabajo interrumpido y consecuentemente improductivo. ${ }^{12}$ Las organizaciones que adoptan el teletrabajo enfrentan principalmente tres desafíos tecnológicos: conectividad, transferencia de información y comunicación ${ }^{3}$. Y las empresas serán más renuentes a adoptar el teletrabajo cuando hay carencias de los recursos requeridos, dado que la efectividad del teletrabajo demanda equipos y software en mayor cantidad, más rápidos y mejores. ${ }^{22}$

Dados los argumentos expuestos, surge la pregunta de investigación: ¿cuáles factores, relacionados con características personales, actitud y aptitud hacia el uso de TIC, tipo de actividades tele-trabajables y recursos tecnológicos disponibles están asociados con la disposición hacia la adopción del teletrabajo, entre los docentes universitarios?

\section{METOdOLOGÍA}

Para abordar la pregunta orientadora se recurre a la construcción de un instrumento y a la aplicación del mismo entre profesores de universidades públicas y privadas, localizadas en la región del Eje Cafetero-Colombia. En este estudio se usa una invitación vía correo electrónico (disponible en: https://docs.google.com/forms/ d/1 nGrWuH6ae8Tapx2YXPM8JxSBWwiOiEnxnrILUYqZ1GA/ viewform?c=0\&w=1Etusp=mail_form_link) explicando el propósito de la encuesta, con un enlace a la misma, y asegurando confidencialidad en las respuestas. La encuesta contiene principalmente preguntas con respuestas cerradas de selección múltiple, las cuales fueron agrupadas en las cinco dimensiones ya referenciadas. Por su parte, la pregunta orientadora apuntaba a deter- minar si, en un contexto hipotético, el profesor universitario encuestado estaría o no interesado en participar en una prueba piloto de teletrabajo mixto, el cual contemplaba tres modalidades de respuesta: sí, quizás y no estaría interesado.

\section{Procedimiento}

A los profesores encuestados se les solicitó indicar su nivel con el que estaban de acuerdo o en desacuerdo con cada uno de los items en una escala de Likert de cinco puntos desde 1 (muy en desacuerdo) a 5 (muy de acuerdo). Pero para el análisis multivariado, y con el propósito de facilitar la interpretación de los datos, se procede a fusionar las modalidades de respuesta en dos, estar de acuerdo (muy de acuerdo y de acuerdo) y no estar de acuerdo (las otras categorías de respuesta). Inicialmente se procede al uso de Análisis de Correspondencias Múltiples (ACM), el cual es una técnica de interdependencia entre variables predominantemente categóricas que tiene por finalidad la reducción de dimensiones mediante la construcción de mapas perceptuales. ${ }^{23}$ Dicha técnica será usada en el presente estudio con el objetivo de establecer los factores que presentan mayor asociación con la disposición manifestada, la indecisión o el no interés por adoptar el teletrabajo entre los profesores universitarios.

De igual forma, y con el propósito específico de determinar cuáles factores son los que más inciden en tener buena disposición hacia adoptar el teletrabajo, se acudirá a la aplicación de la regresión logística, la cual es una técnica especial de Análisis Discriminante caracterizada por su robustez, dado que se apoya en supuestos menos restrictivos y permite el uso de variables predictivas de tipo categórico ${ }^{23}$. Específicamente, en la investigación adelantada se usó la variable respuesta en forma dicotómica, asumiendo los siguientes valores:

$$
Y= \begin{cases}1, & \text { si accedería a teletrabajar } \\ 0, & \text { no estaría interesado / quizás) }\end{cases}
$$

Para evaluar la bondad del ajuste del modelo obtenido, se acudió a la prueba de Hosmer-Lemeshow, el cual plantea como hipótesis nula el que el modelo se ajusta adecuadamente a los datos. Y como estrategia de validación del modelo se acudirá a la obtención de tablas de contingencia que hacen el cruce entre las categorías de respuesta observada contra las categorías de respuesta predicha por el modelo. Los datos de las encuestas diligenciadas fueron registrados y codificados en el software estadístico SPSS versión 18.0. El análisis incluyó la validación de los datos, específicamente el alpha de Cronbach fue usado como indicador de la consistencia interna de los items de la encuesta para asegurar su fiabilidad, la cual arrojó un valor de 0,80 , lo que indica que el instrumento utilizado presenta un buen nivel de fiabilidad. ${ }^{24}$

\section{RESULTADOS Y DISCUSIÓN}

El número total de docentes encuestados fue de 118, de los cuales el $81,4 \%$ eran de universidades públicas y $18,6 \%$ de privadas; $65,3 \%$ hombres y $34,7 \%$ mujeres; el 32,2\% sin hijos, el 25,4\% con un solo hijo, el 33,9\% con dos hijos y el 8,5\% con tres o más hijos, el 78,8\% nacidos antes de 1981 y el 21,2\% nacidos después de 1980. El número de años promedio de experiencia como profesor universitario fue de 15,6 años. En cuanto a los niveles de formación alcanzado se registró que el 5,9\% eran Profesionales, el 11,9\% 
Especialistas, el 53,4\% Magíster y el 28,8\% Doctores. Y a la pregunta sobre si sabía qué era el teletrabajo, los profesores en su mayoria respondieron afirmativamente como queda registrado en la Figura 1.

Figura 1.

Frecuencia de respuestas a la pregunta ¿Sabe usted qué es el teletrabajo?

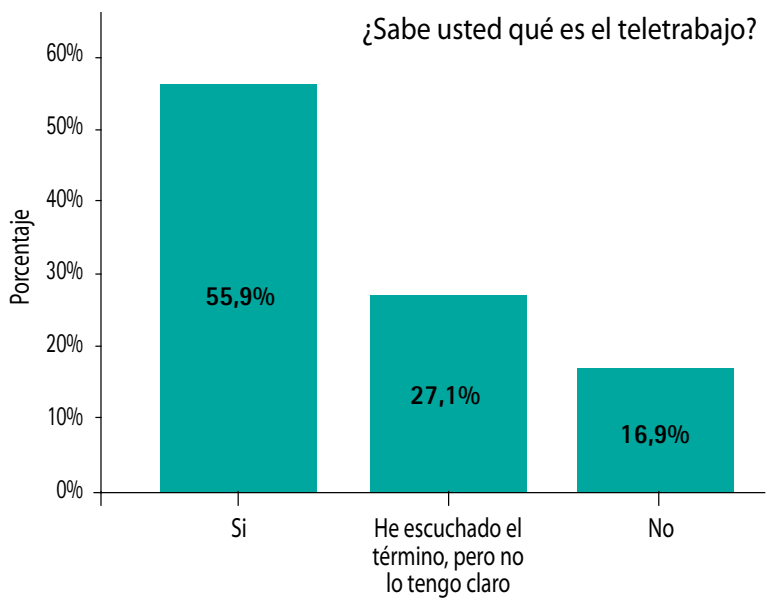

Fuente: reporte descriptivo SPSS v.18

Con relación a la pregunta de interés de la presente investigación sobre el deseo manifestado por los profesores universitarios a participar en una prueba piloto de teletrabajo suplementario, se encontró que en su mayoría expresan disposición por dicha modalidad laboral, dado que el 60,2\% estaría dispuesto a adoptar el teletrabajo en el caso de que se le presentase dicha oportunidad, tal como queda registrado en la Figura 2.

Figura 2.

Frecuencia de respuestas obtenida sobre la disposición a teletrabajar.

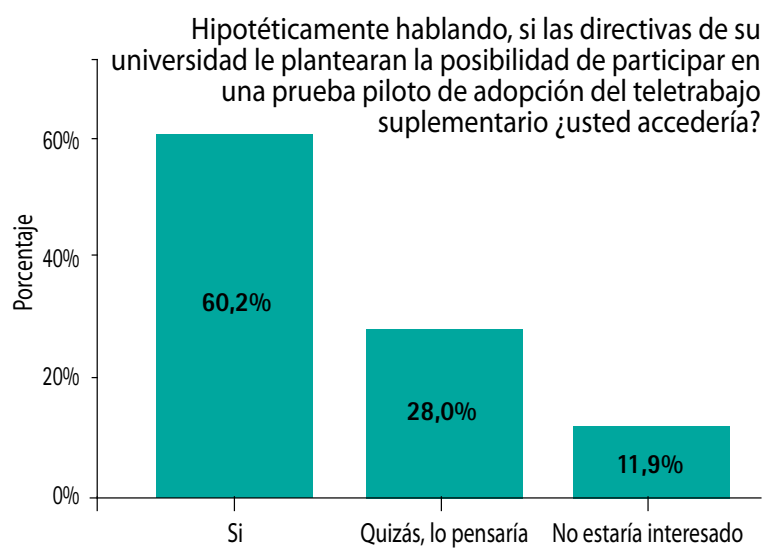

Fuente: reporte descriptivo SPSS v.18

Previo a la realización del análisis multivariado, se procede a filtrar las variables que muestran asociación estadistica con la disposición hacia la adopción del teletrabajo, mediante la prueba chicuadrado de independencia, seleccionándose aquellas con valores $\mathrm{P}$ inferiores a 0,1. La aplicación del procedimiento arrojó que casi ninguna de las variables agrupadas dentro de los aspectos individuales mostró asociación estadística con la disposición a teletrabajar. Específicamente, y a diferencia de lo encontrado en otros estudios $^{2,14,15,18}$, ni el género, ni el grupo generacional, ni el nivel de escolaridad, ni la tenencia de hijos, ni el número de personas con las que se convive, ni el tiempo de movilización al trabajo mostraron asociación con la disposición hacia la adopción por el teletrabajo, pero si el grado de autonomía y flexibilidad percibida por los docentes.

De igual manera, pocas variables relacionadas al tipo de actividades tele-trabajables mostraron asociación con la disposición por teletrabajar. Particularmente, la realización de actividades investigativas y de extensión por fuera de la institución surge como variables explicativas. De manera similar, y en oposición a los hallazgos reportados en otras investigaciones ${ }^{12,22}$, pocas variables agrupadas en los aspectos del entorno laboral surgen como factores influyentes, puntualmente, a excepción de la pregunta que indagaba sobre si el ambiente de trabajo le permitía sentirse que pertenecía a un grupo de trabajo, ninguna de las preguntas conducentes a evaluar las herramientas tecnológicas disponibles para el ejercicio laboral de la docencia mostró asociación estadística con la disposición por teletrabajar.

Contrariamente, y en concordancia con otros hallazgos, ${ }^{10,16,19}$ se localizan las variables agrupadas en los aspectos actitudinales y aptitudinales hacia el uso de las TIC en el ejercicio docente. Ya que son varias, las preguntas relacionadas a los grupos mencionados que muestran asociación con la variable de interés. A saber, preguntas categorizadas en aspectos actitudinales hacia el uso de las TIC en la docencia tales como, el considerar el computador como una herramienta esencial en el ejercicio docente, el que no le incomode tener que estar conectado por fuera del horario de oficina, el considerar las TIC como herramientas primordiales en la enseñanza actual, el afirmar que el uso de las TIC en la docencia necesariamente debe ir direccionado pedagógicamente y el declarar que el aprendizaje enriquecido por las TIC es más efectivo que los enfoques tradicionales, son variables que muestran asociación estadística con el interés manifestado por teletrabajar.

Asimismo, un conjunto de variables agrupadas en aspectos de aptitud hacia el manejo de las TIC, también surgen como variables explicativas de la disposición por teletrabajar. Específicamente, la frecuencia de uso de internet para propósitos académicos y la intensidad de uso de los dispositivos tecnológicos en la realización de actividades relacionadas con el trabajo en horarios no laborales, el tener experiencia en la realización de actividades on-line con los estudiantes, tales como tutorías, evaluaciones, chats, correo electrónico, talleres y trabajos. En igual sentido, surge como otra variable explicativa el hecho de manifestar tener las habilidades requeridas para el uso de las TIC en las actividades laborales cotidianas.

La aplicación del ACM con las variables preseleccionadas, arroja los siguientes resultados. En primera instancia las cargas de ponderación para la variable de interés (interés por teletrabajar) en las tres primeras dimensiones, muestra que esta carga fuertemente en las dos primeras dimensiones y es casi nula en la tercera, 0,459, 0,270 y 0,003, respectivamente, lo que conduce que para el propósito planteado en la investigación, el análisis del primer plano (dimensiones 1 y 2 ) es suficiente. La Figura 3 muestra que la dimensión 1 está fuertemente influenciada por la percepción que se tiene de uso de las TIC en la docencia. Mientras que para la dimensión 2, surge una variable relacionada con la sensación de sobrecarga laboral manifestada por los docentes. Por su parte, se observa que la variable de interés (¿accedería a teletrabajar?) queda bien representada en el plano, cargando a ambas dimensiones. 
Figura 3. Mapa de medidas de discriminación de las variables estudiadas.

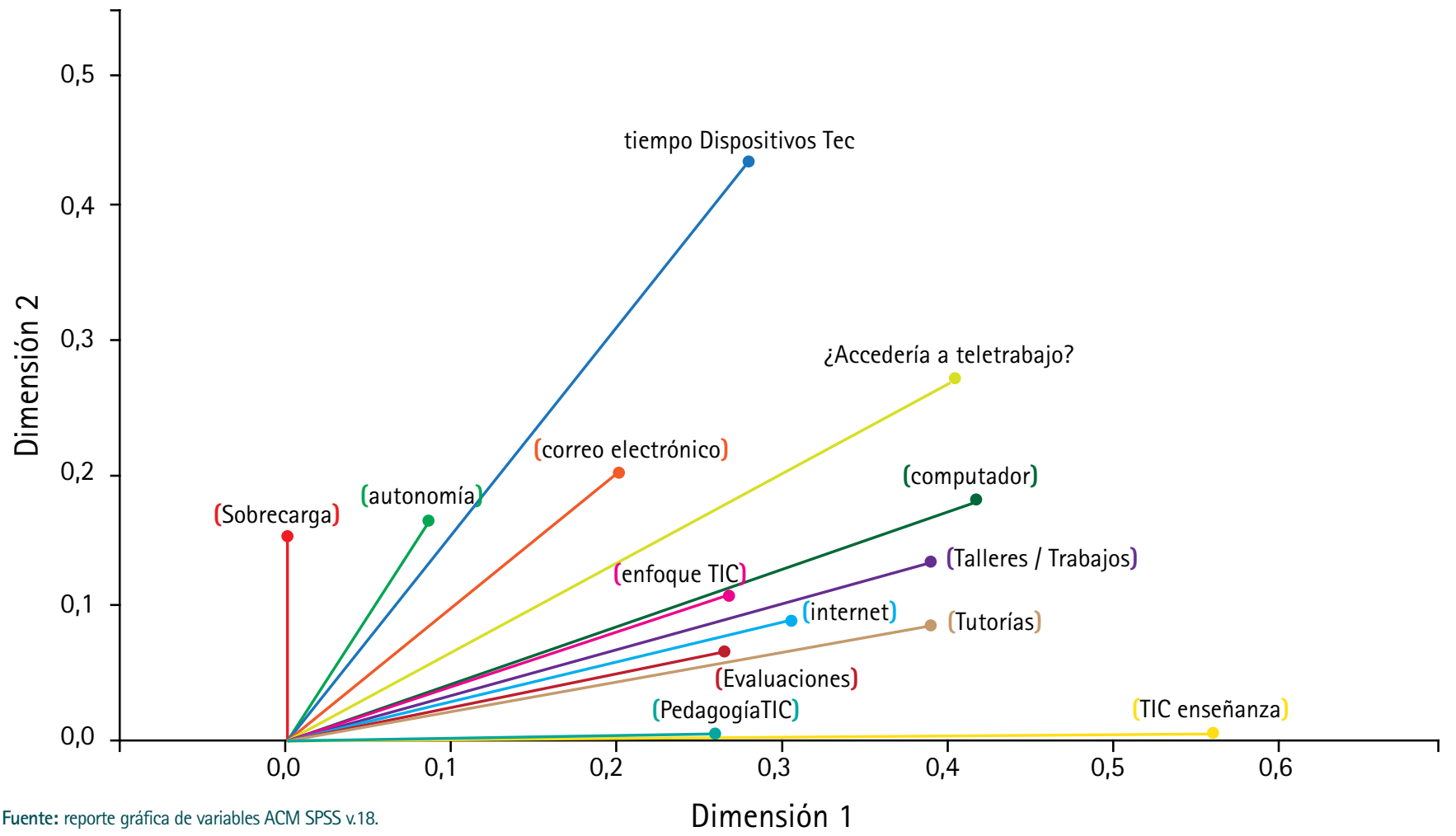

Figura 4. Mapa perceptual del ACM.

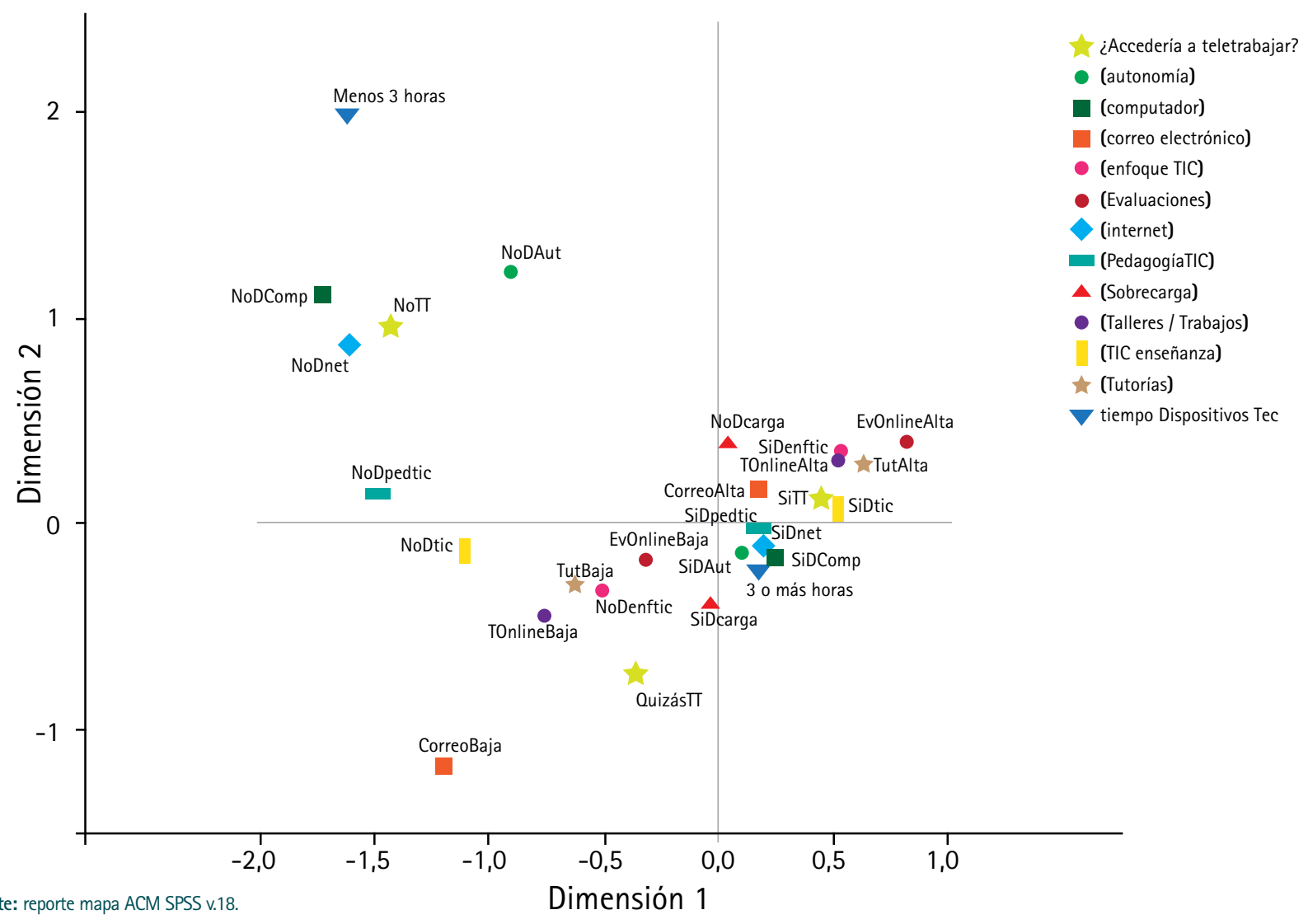


Entre tanto, en la Figura 4 queda plasmado el mapa perceptual obtenido con las variables de estudio. En el mismo se observa que las categorías "si accedería a teletrabajar", "no estaría interesado en teletrabajar” y “Quizás, lo pensaría”, se ubican respectivamente en los cuadrantes cartesianos I, II y III. Puntualmente se observa que la categoría "no teletrabajo" se localiza más distante del origen de coordenadas, lo que es explicado por el hecho de que la misma es la que presenta menos frecuencia de respuestas.

De igual forma, se evidencia la asociación de esta categoría con respuestas tales como el gastar menos de tres horas haciendo uso de los dispositivos tecnológicos en la realización de actividades relacionadas con el trabajo en horario no laboral, el no considerar el computador como una herramienta absolutamente necesaria para el desempeño laboral, el manifestar no usar con mucha frecuencia el internet por fuera de la universidad para propósitos académicos, el declarar no tener la suficiente autonomía para la realización de sus labores y el manifestar no estar de acuerdo con que el uso de TIC en la docencia deba ir direccionado pedagógicamente.

Por su parte, entre las categorías que surgen como diferenciadoras entre tener claridad en manifestar interés por teletrabajar o estar indeciso en ello, están principalmente relacionadas a variables que indagan en aspectos actitudinales hacia el uso de las TIC en la docencia y las que indagan sobre aptitudes en el manejo de las TIC. Específicamente, entre las categorías asociadas a mostrar disposición por teletrabajar, están las relacionadas a aspectos actitudinales como el considerar que las TIC son primordiales en la enseñanza actual y que el aprendizaje enriquecido por las TIC es más efectivo que el de los enfoques tradicionales y el estar de acuerdo con que el uso de las TIC en la docencia debe estar direccionado pedagógicamente o el estar en desacuerdo con la afirmación de que el tiempo no le alcanza para la realización de todas sus actividades laborales.

Entre tanto, aspectos aptitudinales tales como una alta frecuencia de uso en actividades on-line con sus estudiantes muestran asociación con tener disposición a adoptar el teletrabajo.

Por su parte, el estar en desacuerdo con que el aprendizaje enriquecido por las TIC es más efectivo que el de los enfoques tradicionales, y el tener una baja frecuencia de uso en actividades on-line relacionadas con la docencia, así como el considerar que presentan sobrecarga laboral, están asociados a estar indecisos en su intención por adoptar el teletrabajo.

Finalmente, la aplicación de la regresión logística, mediante el método de introducción de variables hacia atrás, es decir, partiendo del conjunto total de variables preseleccionadas, para luego ir eliminando una a una hasta la obtención de un grupo de variables significativas al 95\%, arrojó como resultado que las variables que mejor predicen la disposición hacia adoptar el teletrabajo son las relacionadas con si los docentes consideran o no a las TIC como primordiales en la enseñanza actual, y si están de acuerdo con que el aprendizaje enriquecido por las TIC es más efectivo que el alcanzado con los enfoques tradicionales, tal como queda registrado en la Tabla 1.

Tabla 1.

Variables obtenidas para el modelo de regresión logística.

\begin{tabular}{lccccccc} 
& & & & & \multicolumn{3}{c}{ I.C. 95\%para EXP(B) } \\
& B & E.T. & Wald & Sig. & Exp(B) & Inferior & Superior \\
TIC enseñanza & 1,270 & 0,466 & 7,435 & 0,006 & 3,559 & 1,429 & 8,864 \\
Enfoque TIC & 1,204 & 0,469 & 6,572 & 0,010 & 3,332 & 1,328 & 8,363 \\
Constante & $-0,884$ & 0,344 & 6,613 & 0,010 & 0,413 & &
\end{tabular}

Fuente: reporte regresión logistica SPSS v.18.
De la misma se desprende que el modelo obtenido es,

$$
\begin{aligned}
& \mathrm{P}(\text { adoptar el teletrabajo) } \\
& =\frac{1}{1+e^{(-0,88+1,3 \text { TICenseñanza+1,2EnfoqueTIC })}}
\end{aligned}
$$

De los resultados de la Tabla 1 se desprende que un docente que considere que las TIC son primordiales en la enseñanza actual es 3,6 veces más probable que adopte el teletrabajo que aquel que considera lo contrario. Y aquel que esté de acuerdo con que el aprendizaje enriquecido por las TIC es más efectivo que el de los enfoques tradicionales es 3,3 veces más probable que adopte el teletrabajo que aquel que considera lo contrario. Por su parte, la aplicación de la prueba de ajuste del test de Hosmer-Lemeshow arroja que no se rechaza la hipótesis nula $(P=0,881)$, es decir, el modelo se ajusta de forma adecuada a los datos. Y la tabla de clasificación cruzada mostrada en la Tabla 2, arroja un poder predictivo medianamente bajo, del 72\% de efectividad, aunque mucho mejor si el propósito específico es predecir si el docente universitario adoptará el teletrabajo, con una tasa de aciertos del 80,3\%.

Tabla 2.

\begin{tabular}{|c|c|c|c|c|}
\hline \multirow[b]{2}{*}{ Observado } & & \multicolumn{3}{|c|}{$\begin{array}{l}\text { Pronosticado } \\
\text { Intención por teletrabajar }\end{array}$} \\
\hline & & No/QuizásTT & SiTt & $\begin{array}{l}\text { Porcentaje } \\
\text { correcto }\end{array}$ \\
\hline \multirow{2}{*}{$\begin{array}{l}\text { Intención } \\
\text { por teletrabajar }\end{array}$} & No/Quizas $\Pi$ & 28 & 19 & 59,6 \\
\hline & SiTT & 14 & 57 & 80,3 \\
\hline Porcentaje global & & & & 72,0 \\
\hline
\end{tabular}

Tabla de clasificación cruzada para el modelo obtenido.

Fuente: reporte validación ACM SPSS v.18.

\section{CONCLUSIONES Y RECOMENDACIONES}

En general, se observa que entre los profesores universitarios hay un interés manifiesto por teletrabajar, dado que tan sólo un 11.2\% no estaría dispuesto a hacerlo. Y una posible razón a no estar interesado podría ser el desconocimiento, dado que un 16.2\% de los encuestados reconoció previamente no saber qué era el teletrabajo. Del estudio surge un perfil del profesor que manifiesta no estar interesado en teletrabajar, el cual se caracteriza por hacer poco uso de las herramientas tecnológicas por fuera de la institución, el considerar que el computador no es una herramienta esencial en su actividad laboral, el manifestar no usar con mucha frecuencia el internet por fuera de la universidad para propósitos académicos, el declarar no tener la suficiente autonomía para la realización de sus labores y el manifestar no estar de acuerdo con que el uso de TIC en la docencia deba ir direccionado pedagógicamente.

A la luz de los resultados no se evidenció una influencia significativa entre la mayoría de las variables agrupadas en características individuales, el entorno laboral y el tipo de actividades tele-trabajables que realiza. Pero sí entre gran parte de las variables alusivas a los aspectos actitudinales y aptitudinales hacia el uso de las TIC. Especificamente, muestran una alta asociación con la disposición por adoptar el teletrabajo a aspectos relacionados al uso frecuente de las herramientas TIC en actividades laborales cotidianas tales como el evaluar, el realizar talleres, el dar asesorías o la atención de dudas. En igual sentido, aspectos de percepción hacia la incorporación de las TIC en la docencia, tales como el considerar que 
las TIC son primordiales en la enseñanza actual y que el aprendizaje enriquecido por las TIC es más efectivo que el de los enfoques tradicionales, así como el estar de acuerdo con que el uso de las TIC en la docencia debe estar direccionado pedagógicamente.

La aplicación de la regresión logística arrojó como variables significativas en la perspectiva de predecir la probabilidad de que el profesor universitario adopte el teletrabajo, dos variables de percepción, a saber, el hecho de considerar a las TIC como primordiales en la enseñanza actual y el estar de acuerdo con que el aprendizaje enriquecido por las TIC es más efectivo que el de los enfoques tradicionales.

Entre las recomendaciones sugeridas, están la de ahondar en aspectos poco explorados en el presente estudio, tales como las diferencias asociadas a la naturaleza del institución, pública o privada, la incidencia de los conflictos familia-trabajo, entre otros, para lo cual sería necesario incrementar el esfuerzo de muestreo en la búsqueda de representatividad para la generalización de los resultados.

\section{Agradecimientos}

Los autores agradecen a la Universidad Nacional de Colombia sede Manizales a través de la dirección de investigaciones (DIMA) por el apoyo institucional y financiero al desarrollo de esta investigación (proyecto: "Limitaciones y barreras de los empresarios manizaleños frente a la adopción del teletrabajo en sus empresas" código Hermes 28596).

\section{REFERENCIAS}

1. Baruch Y. Teleworking: benefits and pitfalls as perceived by professionals and managers. New Tech Work Employ. 2000;15(1):34-49.

2. Pinsonneault $A$, Boisvert $M$. The Impacts of Telecommuting on Organizations and Individuals: A review of the literature. In: Jhonson N, editor. Telecommuting and Virtual Offices.: Issues and Opportunities. Pennsylvania: IGI; 2001. p.162184.

3. Pearce II J. Successful Corporate Telecommuting with Technology Considerations for Late Adopters. Organ Dyn. 2009;38(1):16-25.

4. Kossek E, Lautsch B, Eaton S. Telecommuting, control, and boundary management: Correlates of policy use and practice, job control, and work-family effectiveness. J Vocat Behav. 2006; 68(2):347-367.

5. Golden T, Altering the Effects of Work and Family Conflict on Exhaustion: Telework During Traditional and Nontraditional Work Hours. J Bus Psychol. 2012; 27(3):255-269.

6. Nilles $J$ et al. Telecommuting-An Alternative to Urban Transportation Congestion. IEEE T Sys Man Cyb. 1976; 6 (2): 77-84.

7. Burbach M, Day F. Does Organization Sector Matter in Leading Telework Teams? A Comparative Case Study. International J Bus Inform Technol. 2012;1(1):29-42.

8. Bonilla L, Plaza D, Soacha De Cerquera G, Riaño-Casallas M. Teletrabajo y su Relación con la Seguridad y Salud en el Trabajo. Cienc Trab. 2014;16(49):38-42.

9. $\mathrm{Ng} \mathrm{Ch}$. Academics Telecommuting in Open and Distance Education Universities: Issues, challenges, and opportunities. IRRODL. 2006;7(2):1-16.

10. Baelo R. Satisfacción del profesorado universitario con la integración de las Tecnologias de la Información y la Comunicación (TIC). Etic@net. 2011;9(11):253-276.

11. Tustin D. Telecommuting Academics within an Open Distance Education Environment of South Africa: More Content, Productive, and Healthy?. IRRODL. 2014;15(3):185-214.

12. Illegems $V$, Verbeke $A$, S'Jegers R. The organizational context of teleworking implementation. Technol Forecast Soc. 2001; 68(1):275-291
13. Dickson K, Clear F. Management Issues in the Adoption of Telework Amongst SMEs in Europe. PICMET 2006 Proceedings, 9-13 Jul, Istanbul, Turkey. New Jersey: IEEE; 2006. p.1703-1708.

14. Henquinet J. Telecommuter selection: A systems perspective. Telecommuting and Virtual Offices: Issues and Opportunities. In: Jhonson N, editor. Pennsylvania: IGI; 2001. p.118-129.

15. Nicholas A, Guzmán I. Is Teleworking for Millennials? Digital Commons @ Salve Regina. 2009;1(1):00-12.

16. Bailey $D$, Kurland N. A review of telework research: finding, new directions, and lessons for the study of modern work. J Organ Behav. 2002; 23(1):383-400.

17. Singh P. et al. On modeling telecommuting behavior: option, choice, and frecuency. Transportation. 2013;40(1):373-396.

18. Peters $P$, Tijdens K. Wetzels $C$. Employees' opportunities, preferences, and practices in telecommuting adoption. Inform Manage. 2004; 41:469-482.

19. Rienties B, Brouwer N, Lygo S. The effects of online professional development on higher education teachers' beliefs and intentions towards learning facilitation and technology. Teach Teach Educ. 2013; 29(1):122-131.

20. Kirkwood A, Price L. Adaptation for a Changing Environment: Developing learning and teaching with information and communication technologies. IRRODL. $2006 ; 7(2): 1-14$

21. Lagunes $A$ et al. Comparativo del uso de Tecnologias de la Información y Comunicación (TIC) por profesores de dos universidades públicas de México. Form Univ. 2015;8(2):11-18.

22. Davis $A_{1}$. Telework productivity and effectiveness: factors that influence results oriented job assessments [on line] Oregon: University of Oregon; 2011 [cited nov 19 2013]. Disponible en: https://scholarsbank.uoregon.edu/xmlui/bitstream/ handle/1794/11385/Davis-2011.pdf?sequence=1.

23. Díaz LG, Estadistica Multivariada. Bogotá: Universidad Nacional de Colombia; 2002.

24. Raykov T. On the Use and Utility of the Reliability Coefficient in Social and Behavioral Reseach. Qual Quant. 2001;35: 253-263. 